\title{
Upper Lip Rejuvenation by Myocutaneous Subnasal Lift Technique
}

\author{
NADA A. MAHMOUD, M.D. and KARIM S. MASSOUD, M.D. \\ The Department of Plastic, Burns and Maxillofacial Surgery, Faculty of Medicine, Ain Shams University, Cairo, Egypt
}

\begin{abstract}
Background: With aging, the upper lip gradually loses its youthful pout and slowly droops downwards.

Aim: In this study, we present a modification to the subnasal lip lifting technique to improve scar quality and aesthetic outcome.

Patients and Methods: This study included twenty-three adult female patients. Elliptical subnasal excision including skin and muscle was done. Assessment criteria included scar quality, lip height, vermillion and incisor show. In some cases, objective measurements of the lip was used.

Result: All patients had accepted hidden scar. Total lip height and prolabium length were significantly decreased and vermilion height increased. One case had minimal change in the vermilion show, improved with filler injection. Finally, all patients showed overall satisfactory outcome.
\end{abstract}

Conclusion: A wedge excision form the superficial part of the orbicularis oris muscle with skin excision reduces tension on the scar of subnasal upper lip lift. The excision does not affect upper lip muscle function and it can improve the aesthetic outcome and achieve a younger pleasant look. Larger patient population studies are needed to confirm the data. Our proposed preliminary objective assessment method is a very interesting method that can validate future results.

Evidence based medicine: Level IV, case series.

Key Words: Upper lip - Lip rejuvenation - Lip aesthetics Subnasal lip lift.

\section{INTRODUCTION}

The lips are the central feature in the lower third of facial aesthetics. The aging upper lip gradually loses its youthful pout and slowly droops downwards with the appearance of vertical wrinkles [1].

Gravity causes stretch of the upper lip skin, resulting in increased vertical height of the prolabium, flattening of the philtrum, inversion and horizontal flattening of the vermilion, and excessive hooding of the upper incisors [2]. Austin supposed that hooding of the upper incisor may be the key for this distracting problem. Therefore, shortening of the upper lip may lead to dramatic change in patients' appearance [3].

The orbicularis oris muscle is also affected by the aging process. The muscle fibers lose their elasticity, which are partly replaced by connective tissue and becomes thinner. A deterioration of the dentition and bony atrophy may be added [4]. These changes appear in profile view as loss of the concave curvature of the upper lip.

In 1971, Cardoso and Sperli [5] first presented the cuneiform resection of a strip of skin at the nasolabial junction. Rozner and Isaacs [6], as well as Fonseca [7] who recommended skin excision with downward undermining. Later, many techniques were published to decrease the elongated white lip, either through horizontal excision along the vermilion border [8] or in the form of $v-y$ advancement [9]. Austin popularized the subnasal lip lifting technique [3]. It has been used with different skin patterns such as wavy ellipse or bullhorn with or without undermining [10].

In this study, we modify sub-nasal lip lifting through excising a wedge from the superficial part of the underlying orbicularis oris muscle; aiming for tension free closure, better scar quality and improving the aesthetic outcome.

\section{PATIENTS AND METHODS}

This study included twenty-three female patients complaining of aging long lip in the period from June 2014 to December 2016. Seventeen patients underwent myocutaneous lip rejuvenation during face lift procedure.

Surgical Technique Fig. (1): The amount of excess skin that need to be excised was determined by pushing the lip upwards till the desired amount of incisor show is achieved (about $2 \mathrm{~mm}$ ). The incision began at the alar crease and traversed to the other ala following the subcolumellar-lip border 
bilaterally (ellipse shaped incision). Then, a $4 \mathrm{~mm}$ wedge was excised from the upper part of Orbicularis oris muscle just below the columella. Muscle closure was performed using 3 stitches of 4/0 PDS; the central stitch is attached to the periosteum of the anterior nasal spine. After hemostasis, the wound was closed using simple interrupted 6-0 Prolene sutures.

Post-operatively, patients received oral antiedema agents for one week. Stitches removed after one week.

Assessment of surgical outcome was done clinically by the surgical team. In addition, preoperative and post-operative photographs were analyzed by an independent plastic surgeon. Scar assessment criteria were adopted from the PatientT and Observer Scar Assessment Scale (POSAS) and
The Stony Brook Scar Evaluation Scale [11,12]. The assessment criteria included scar height, width, erythema, pigmentation, pliability, pain, itching or pruritus interfering or not with quality of life, and scar visibility to patient's relatives.

In addition to scar assessment, aesthetic outcome of the technique was evaluated subjectively using lip height, vermilion show and incisor show.

To augment subjective assessment, we started to measure lip height pre-operatively and postoperatively in the last 8 cases in our series aiming at objective assessment. We marked three vertical points (A, B, and $\mathrm{C}$ ) as reference points to measure the length of the prolabium and vermilion height pre and post-operatively (Fig. 2). These measurements were taken pre-operatively, immediate postoperative and 12 months post-operative.
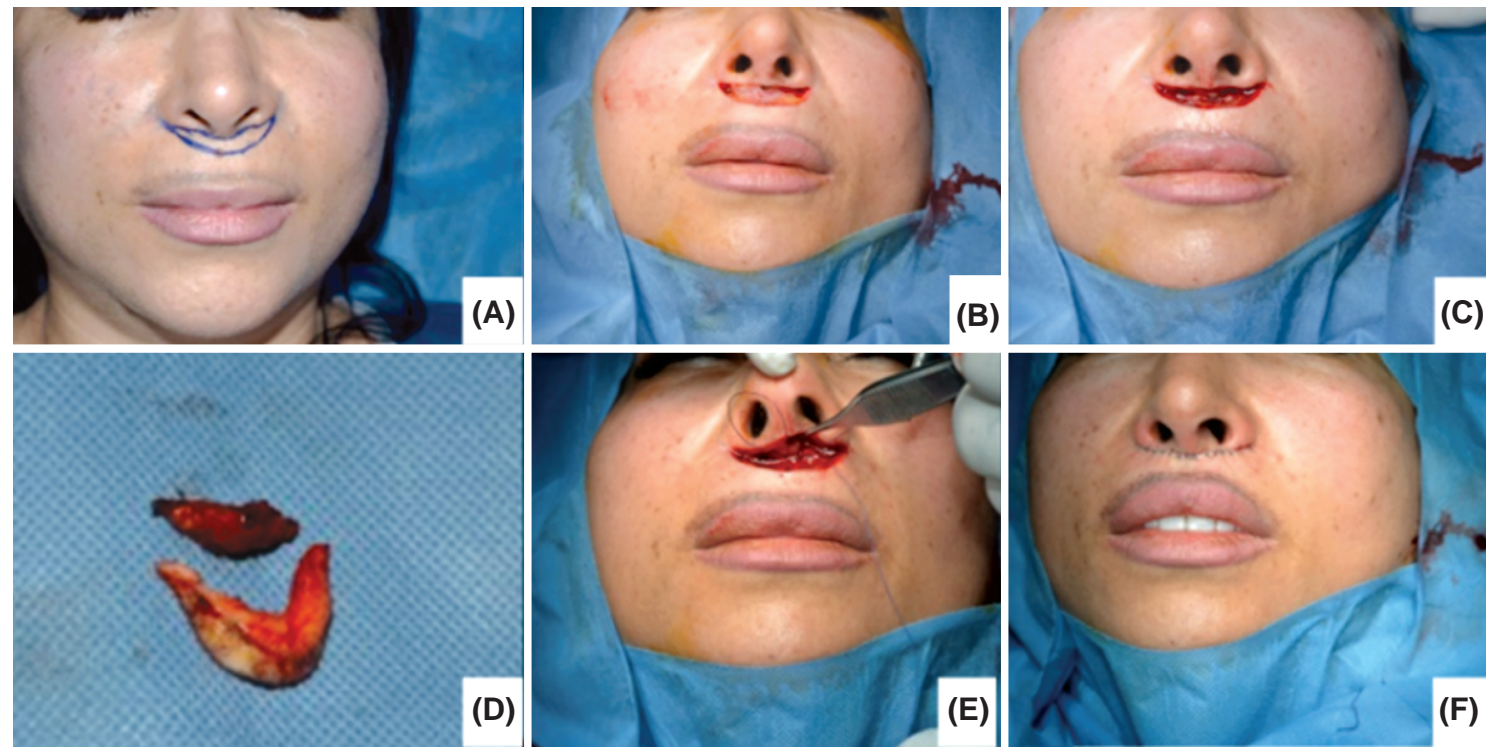

Fig. (1): Surgical technique (A) Pre-operative marking, (B-D) Excision of skin then muscle, (E-F) Closure of muscle layer then skin showing improvement of incisor and vermilion show.

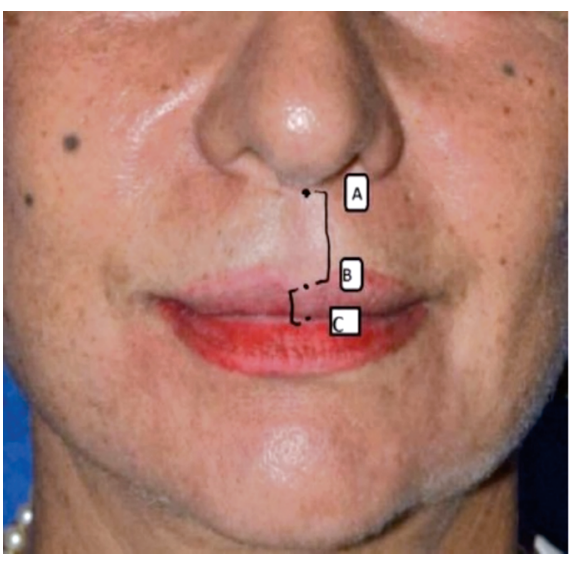

Fig. (2): Points to detect vertical lip measurements. Point A (center of the base of columella), point B (center of the base of cuboids bow), point $C$ (center of the line between dry and wet vermilion). Prolabium height was measured from point $\mathrm{A}$ to $\mathrm{B}$, vermilion height from point $\mathrm{B}$ to $\mathrm{C}$.

\section{RESULTS}

Patients' age ranged between 47-65 years with a mean of 53.8 years. The follow-up period ranged from 10-16 months (mean was 12.1 months).

Scar assessment revealed that all patients had good results with well-hidden scar appearance. No patient experienced hypertrophic scaring, widened scars, or scar pigmentation. No associated symptoms such as pain or pruritus were recorded. All scars were nearly not felt by 3-6 months postoperatively. Regarding scar visibility, no patient had visible attracting scar, only 2 patients had visible scar to patient's relatives from near distances, and 21 patients had nearly non-visible scars even from near distances. 
Satisfactory aesthetic outcome could be achieved in all patients except one patient. Analysis of pre and post-operative photography show marked improvement with shortening of the prolabium height which was occurred in all patients and could be maintained in the late post-operative period (12 months post-operative). Vermilion height and in- cisor were moderately improved and also maintained with minimal changes in late post-operative. There were minimal clinical changes between early and late post-operative aesthetic outcome. The only patient with non-satisfactory result needed lip volume augmentation using fillers to achieve satisfactory result (Fig. 3).
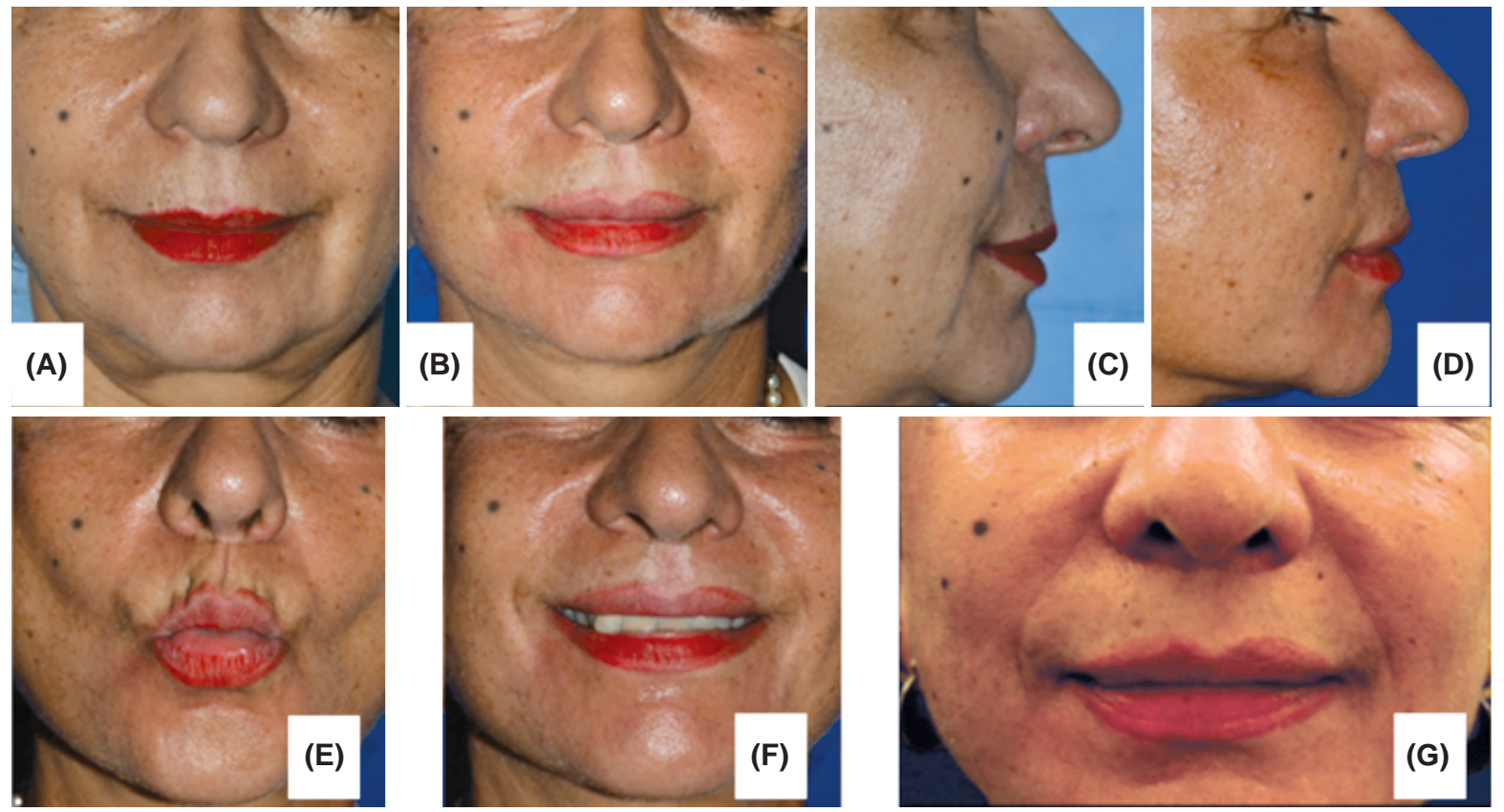

Fig. (3): (A,C) Pre-operative photo in anterior and lateral view respectively, (B,D) Post-operative photo in anterior and lateral view show shortening of the white upper lip and increase in vermilion show. (E,F) Show normal muscle function. (G) Show non-apparent scar.

No major complications recorded in any patients. Transient scar erythema recorded in few patients. Mild paresthesia occurred and resolved spontaneously by 2 weeks post-operative. Neither nasal synechia nor external valve airway problems were recorded. No orbicularis oris muscle dysfunction occurred.

Pre and post-operative measurements detect a statistically significant change in both vertical lip measurements between pre-operative and postoperative measurements. There is statistically nonsignificant difference between early and late postoperative measurements. The prolabium length was decreased in all patients by about $20 \%$ while vermilion height increased by about $18 \%$ except in three cases where no significant change was recorded (Tables 1,2).

Table (1): There is statistical significant difference between the mean of pre-operative and early post-operative vertical upper lip measurements.

\begin{tabular}{lllcc}
\hline & Control & $\begin{array}{c}\text { Mean pre- } \\
\text { operative }\end{array}$ & $\begin{array}{c}\text { Mean early } \\
\text { post-operative }\end{array}$ & $\begin{array}{c}p \text { - } \\
\text { value }\end{array}$ \\
\hline Prolabium length & $13.78 \mathrm{~mm}$ & $18.8 \mathrm{~mm}$ & $14.11 \mathrm{~mm}$ & 0.0001 \\
Vermilion height & $6.20 \mathrm{~mm}$ & $5.3 \mathrm{~mm}$ & $5.92 \mathrm{~mm}$ & 0.0001 \\
\hline
\end{tabular}

Table (2): There is no statistical significant difference between the mean of early and late post-operative vertical upper lip measurements.

\begin{tabular}{lccc}
\hline & $\begin{array}{c}\text { Mean early } \\
\text { post-operative }\end{array}$ & $\begin{array}{c}\text { Mean late } \\
\text { post-operative }\end{array}$ & $\begin{array}{c}p^{-} \\
\text {value }\end{array}$ \\
\hline Prolabium length & $14.11 \mathrm{~mm}$ & $14.96 \mathrm{~mm}$ & 0.0220 \\
Vermilion height & $5.92 \mathrm{~mm}$ & $6.23 \mathrm{~mm}$ & 0.035 \\
\hline
\end{tabular}

\section{DISCUSSION}

Aging of the upper lip has three main problems: Wrinkles, thinning, and lengthening. The traditional face lift procedures do not address these aging features. Many ancillary procedures such as resurfacing either by laser, dermabrasion, and chemical peeling have described. In addition, fillers were used to improve the fine wrinkles and thinning of the upper lip. However, face lift and ancillary procedures cannot solve the problem of upper lip lengthening.

Multiple surgical techniques were described to address the elongated upper lip through different lip lifting procedure; direct vermillion advancement [8], V-Y advancement [9], and subnasal lip lift [3] 
were used. On the other hand, Tolbert Wilkinson [13] advised to put lip shortening procedures at rest claiming that any benefit gained can't be weighed with the resulting scar.

Direct vermillion advancement improves vertical lip height but attenuates the lip roll. In addition, the natural "white roll" is obliterated by a flat permanent apparent scar along the red: White border. Moreover, it disturbs the central aesthetic components of the upper lip [8,10]. Some surgeons recommend this technique for the lateral lips [14].

The V-Y advancement technique was reported through intraoral incisions; however, the results were unpredictable. The prolonged post-operative edema is another reported disadvantage of this technique [15].

The subnasal lip lift provides a predictable increase in the vertical height of the red lip. It also preserves the delicate anatomy at the lip border and improves the red: White lip ratio in a predictable fashion [10].

Sub-nasal excision has different skin patterns such as wavy ellipse or bullhorn with or without undermining. Small vertical line incision was used to decrease widening of the philtrum or flattened Cupid's bow. Bilateral L-shaped excision along the vertical limb of the philtrum with vertical or horizontal plication of the orbicularis muscle was also described [3]. All these techniques achieved upper lip shortening, however, the gain of excellent or non-apparent scar still had many controversy among these techniques. In addition, the actual amount of skin excision still had variable estimations among these techniques; either 3 to $4 \mathrm{~cm} \mathrm{[4]}$ or one third to one half of the prolabium or 6 to $10 \mathrm{~cm}$ as some believed that over correction will be needed [2], but most of surgeons believe that excision is variable and they think that about one third of the total lip height maybe needed [1].

Despite different skin patterns in sub-nasal lift, none of them deal with the muscle lengthening. Santanché and Bonarrigo were the first to perform muscle excision. They excised a small portion from the cranial part of the muscle through a sub alar incision to shorten the prolabium. Sometimes, they used the excised part as a graft to augment the lower vermilion if needed [16].

From our point of view, we think that muscle lengthening must be addressed in lip lengthening procedures for different reasons; firstly, it is considered a part of the pathology of aging of the lip.
Secondly, removal of a wedge from the muscle can aid in tension free closure. Thirdly, wedge excision can help to evert lip and improve vermilion show. Finally, no subcutaneous dissection is carried on which prevent any distortion in philtrum ridges.

In our technique, we shortened the upper lip through subnasal excision of approximately $20 \%$ of the prolabium length (about 4-5mm) according to each case. The wedge resection extends to the superficial part of the orbicularis oris muscle. Then, suturing the muscle into anterior nasal spine serves for lip elevation and to maintain long term results. Tension free repair after muscle resection help in un-eventual wound healing and concealed scar. We avoid any disruption of the deep component of the orbicularis oris muscle to avoid any muscle dysfunction. It is very important to mention that the amount of skin resection is fundamental to obtain good scar. Muscle excision cannot compensate for over resection of the skin.

Our technique is different from that described by Santanche' and Bonarrigo. Their incision is starting in alar lateral extending to nostril and sparing columella. Although, the scar is hidden, it can cause nostril distortion of nostril or synechiae. They perform subcutaneous dissection while in our technique, we avoid any dissection to avoid any lip distortion.

The subjective evaluation of the aesthetic outcome of the technique showed very satisfactory results. Lip shortening improves vermilion height, and incisor show that gives a dramatic change in patients' look. The preliminary data obtained from objective measurements confirmed these clinical findings, however, the sample size is very small to give conclusive findings.

The myocutaneous lip lifting could achieve a good aesthetic outcome that could be maintained. The tension free repair shared in improvement of scar quality that yielded to nearly hidden scar.

\section{Conclusion:}

A wedge excision from the superficial part of the orbicularis oris muscle with proper amount of skin excision reduces tension on the scar of subnasal upper lip lift. The excision does not affect upper lip muscle function and it can improve the aesthetic outcome and achieve a younger pleasant look. Larger patient population studies are needed to confirm the data. Our proposed preliminary objective assessment method can validate future results. 


\section{REFERENCES}

1- Hinderer U.T.: Ageing of the upper lip: A new treatment technique. Aesthetic Plast. Surg., Nov.-Dec., 19 (6): 51926, 1995.

2- Waldman S.R.: The Subnasal Lift. Facial Plast. Surg. Clin. N. Am., Nov., 15 (4): 513-6, viii, 2007.

3- Austin H.: The lip lift. Plastic and Reconstructive Surgery Vol.: 77, No: 6: 990994, 1986.

4- De la Plaza R. and De La Cruz L.: Can some facial rejuvenation techniques cause iatrogenia? Aesth. Plast. Surg., 18 (2): 205, 1994.

5- Cardoso A.D. and Sperli A.E.: Rhytidoplasty of the upper lip In: Hueston J.T. (ed): Transactions of the Vth International Congress of the PRS. Melbourne: Butterworth, 1971, 34: 481, 1981.

6- Rozner L. and Isaacs G.L.: Lip lifting. Br. J. Plast. Surg., 34: 481, 1981.

7- Fonseca J.L.: Aesthetic reduction of the upper lip. Ann. Plast. Surg., 19 (5): 400, 1987.

8- Felman G.: Direct upper lip lifting: A safe procedure. Aesthet. Plast. Surg. J., 17: 291-295, 1993.
9- Lassus C.: Thickening the thin lips. Plast. Reconstr. Surg., 68: 950, 1981.

10- Ali M.J.1, Ende K. and Maas C.S.: Perioral Rejuvenation and Lip Augmentation. Facial Plast. Surg. Clin. North Am., Nov., 15 (4): 491-500, vii, 2007.

11- Draaijers L.J., Tempelman F.R., Botman Y.A., et al.: The Patient and Observer Scar Assessment Scale: A reliable and feasible tool for scar evaluation. Plast. Reconstr. Surg., 113: 1960-65, 2004.

12- Singer A.J., Arora B., Dagum A., et al.: Development and validation of a novel scar evaluation scale. Plast. Reconstr. Surg., 120 (7): 1892-7, 2007.

13- Wilkinson T.S.: Lip lift resection. Plast. Reconstr. Surg., Jul., 94 (1): 212, 1994.

14- Weston G.W., Poindexter B.D., Sigal R.K. and Austin H.W.: Lifting lips: 28 years of experience using the direct approach to rejuvenating the aging mouth. Aesthet. Surg. J., Mar.-Apr., 29 (2): 83-6. Doi: 10.1016/j.asj.2009.01.013, 2009.

15- Ho L.C.: Augmentation cheiloplasty. Br. J. Plast. Surg., Jun., 47 (4): 257-62, 1994.

16- Santanché P. and Bonarrigo C.: Lifting of the upper lip: Personal technique. Plast. Reconstr. Surg., May, 113 (6): 1828-35; discussion 1836-7, 2004. 